\title{
Acute Sensorimotor Polyneuropathy as an Early Sign of Periarteritis Nodosa: Report of a Case
}

\author{
Valery Kazakov $^{* 1,2}$, Dmitry Rudenko ${ }^{1,2}$, Tima Stuchevskaya ${ }^{1,2}$, Oxana Posokhina $^{1,2}$, Semen Perfilyev $^{1}$ and \\ Alexander Skoromets ${ }^{1}$ \\ ${ }^{1}$ Department of Neurology, First Pavlov State Medical University, Russia \\ ${ }^{2}$ Neurological Department of City Hospital 2 of St. Petersburg, Russia
}

*Corresponding author: Valery Kazakov, Professor of Clinical Neurology, Department of Neurology, First Pavlov State Medical University of St. Petersburg, L. Tolstoy Str. 6/8, St. Petersburg, Russia.

Received Date: June 01, 2019

Published Date: June 10, 2019

\section{Abstract}

We examined a patient aged 31 who had a sudden burning paraesthesia, pain and numbness in the lower legs together with an increased temperature of $39^{\circ} \mathrm{C}$. Clinical examination showed asymmetrical sensory polyneuropathy more clearly seen in the lower legs and the left wrist, with high ESR (up to $44 \mathrm{~mm} / \mathrm{h}$ ), leukocytosis, slight anemia and proteinuria. CSF was normal. After three weeks the temperature suddenly increased again up to $39^{\circ} \mathrm{C}$ and severe flaccid distal tetraparesis was seen more clearly with foot drop in the left lower leg and dense oedema in the left wrist, purple cyanosis and haemorrhagic foci appeared on the skin of the toes, feet, lower legs and left wrist. ESP increased up to $65 \mathrm{~mm} / \mathrm{h}$, CK was 200 IU (normal=190 IU) and hypergammaglobulinaemia developed. An EMG study showed sensorimotor, mainly axonal, polyneuropathy with different degrees of involvement of some nerves and with conduction block in the left ulnar nerve. Muscle biopsy revealed findings of inflammatory vasculitis that resembled polyarthritis nodosa with secondary denervation atrophy and non-specific myositis.

The patient was treated with high doses of prednisolone, dexamethasone and cyclophosphamide with plasmapheresis. Motor disturbances and pain decreased and the patient began walking with a stick. However, the necrosis of the toes gradually progressed to dry gangrene and amputations of the toes were carried out three months after the disease began. At that time the patient had the clinical features of multisystem disease with progressive heart, lung, liver and kidney failure. The patient died suddenly of pulmonary artery thrombo-embolism a year after the onset of the disease. An autopsy confirmed the diagnosis of polyarteritis nodosa ( $\mathrm{PH}$ ). Thus, in this patient the asymmetrical sensory polyneuropathy progressed rapidly in symmetrical sensorimotor peripheral polyneuropathy which preceded the clinical multisystem involvement in polyarteritis nodosa.

Keywords: Asymmetrical sensory polyneuropathy; Burning paraesthesia; Necrosis of the toes; Progressive heart; Lung; Liver and kidney; Polyarteritis nodosa

\section{Introduction}

Periarteritis nodosa (PN) is one of the systemic necrotic vasculitis. The characteristic pathological feature is inflammation and necrosis of vascular walls of small and middle sizes arteries. These changes are the basis for the infarction and ischemia which can be seen in different tissues and organs. Lesion of the nervous system occurs in $50 \%$ of cases with PN [1]. The classical example of a peripheral nervous system lesion in PN is mononeuritis multiplex or asymmetrical peripheral motorsensory polyneuropathy. Often neurological signs appear after polyorganic pathology in PN patients, but in some cases lesions of peripheral or central nervous systems may be the first sign of the disease [2-4].

\section{Case Report}

The patient (a man) aged 31 was admitted to the Neurological Department complaining of burning pains and numbness of the distal parts of the legs, and difficulty in walking due to severe pain. His temperature rose to $39^{\circ} \mathrm{C}$ and symptoms of nasal congestion appeared. 4 day after the onset he was hospitalized with the diagnosis of Guillain-Barre Syndrome. But 2 weeks after admission his general condition deteriorated with increasing neurological abnormalities. His temperature suddenly increased again to $39^{\circ} \mathrm{C}$. Pain burning and numbness increased in the legs and some symptoms appeared in the hands. He complained of asymmetrical 
weakness of the distal parts of the legs and small hand muscles, worse on the left. Marked oedema of feet and toes appeared which spread over the lower legs. There was a change of skin of feet toes and lower legs appeared.

\section{Status}

The pulse was 96, the blood pressure increased to $160 / 90$ mmHg. He was normally orientated in place and time. Cranial nerves were normal. Chest and abdomen were normal. The flexors of the neck were spared. All the small muscles of the hand were severely atrophied and moderate atrophy the distal part of forearms was seen. Chest and abdomen were normal. Muscular strength [5]: shoulder girdle, deltoid, upper arms and extensors of the wrists, grade 5; extensors digitorum communis and flexors of the wrists, grade 3 ; interossei volaris and dorsalis and lumbricales, grade 1-2 with both side involved, more clearly on the left. Muscle tone in the upper limbs was decreased. Deep reflexes of the arms were reduced. Abdominal reflexes were absent. There was moderate atrophy of the thigh and severe atrophy of shin muscles. Proximal muscle power including hip and knee flexion and extension were normal. There was no movement of the ankle and toes on the left and power was reduced to 1-2 grades on the right. Muscle tone and knee reflexes were reduced. The ankle jerks and plantar responses were absent on both sides.

There was dysesthesia, hyperpathia and hyperesthesia of all kinds of sensation in the toes, feet and lower third of the lower legs. Lasseg sign was positive. Palpation of ulnar, radial and especially peroneal communis, tibial and dorsal pedis nerves was painful. Palpation of arm and leg muscles was painless. Dense oedema of the lower legs and feet was worse on the left. Purple cyanosis and hemorrhagic foci were seen on the skin of the feet, lower legs, back of the hands and distal part of the left arm. There were necrotic changes of the distal phalanges of the first and third toes of the left foot.

Blood analysis showed a decrease of $\mathrm{Hb}$ to $117 \mathrm{~g} / \mathrm{l}$ and erythrocytes to $3.5 \times 10 / 1$, moderate leukocytosis up to $9.3 \times 10 / 1$. ESP increased to $69 \mathrm{~mm} / \mathrm{h}$. The blood levels of sugar, bilirubin, creatine, urea, cholesterol, serum protein, potassium, calcium and phosphorus were within normal limits, alpha- 1 and alpha-2 globulins were increased up the normal levels. Blood level of SGPT and SGOT were increased up to $97 \mathrm{U} / \mathrm{I}$ (normal less than $43 \mathrm{U} / \mathrm{I}$ ) and $156 \mathrm{U} / \mathrm{I}$ (normal less than $34 \mathrm{U}$ ); and CK was greatly increased up to $2190 \mathrm{U} / \mathrm{I}$ (normal below $190 \mathrm{U} / \mathrm{I}$ ). There was proteinuria$0.39 \mathrm{~g} / \mathrm{l}$.

ECG showed a sinus tachycardia of 110 per min. Fibrogastroscopy was normal. Repeated chest X-ray radiography showed signs of slight oedema of the lungs and possible pericarditis. Ultrasonography showed slight hepatosplenomegaly. Needle EMG (gastrocnemius, tibialis anterior, extensor digitorum communis and abductor pollicis brevis muscles) showed many fibrillation and single/moderate fasciculation potentials, with positive sharp waves at rest. Many long duration and increased amplitude of MUPs appeared on minimal voluntary contraction. On maximum contraction a discrete pattern was evident. $25-33 \%$ of the action potentials were polyphasic. This EMG finding revealed a neurogenic process. On electric stimulation of the saphenous and afferent fibres of median nerves the motor responses were absent. Sensory right ulnar nerve conduction velocities were greatly decreased in distal and mildly slowed in proximal (14 and $44 \mathrm{~m} / \mathrm{sec}$, respectively). Terminal latency was markedly increased up to $7.9 \mathrm{msec}$.

Motor nerve conduction velocities in the distal parts of right and left ulnar nerves were mildly slowed (44 and $38 \mathrm{~m} / \mathrm{sec}$, respectively), but in the proximal parts of ulnar and in the distal and proximal parts of the median nerves were within normal limits. The terminal latencies were increased (in median nerves 6.6 and $4.3 \mathrm{~m} / \mathrm{sec}$, and in the ulnar nerves $3 / 7$ and $4 \mathrm{~m} / \mathrm{sec}$ ). The compound muscle action potentials (CMAPs) were markedly decreased in amplitude on stimulation of the ulnar ( 1 and $0.1 \mathrm{mV}$ ) and median $(0.5$ and $1.2 \mathrm{mV}$ ) nerves. On stimulation of the right peroneal nerve the very lower amplitude $(0.7 \mathrm{mV})$ response was seen only in the tibial anterior muscle. Motor conduction velocity was greatly decreased to $24 \mathrm{~m} / \mathrm{sec}$ (below fibular head-popliteal fossa).

Left motor ulnar nerve conduction showed partial conduction block between the elbow and wrist. The amplitude of the CMAPs was reduced by $79 \%$ and the area by $88 \%$. The criterion for partial conduction block was a $50 \%$ or greater reduction in amplitude and a $40 \%$ or greater reduction in negative peak area of surfacerecorded CMAPs obtained with proximal compared with distal stimulation of ulnar nerve, in the absence of increased duration of CMAPs more than $20 \%$, according [3].

Table 1: Electrophysiological data in the affected motor nerves.

\begin{tabular}{|c|c|c|c|c|c|}
\hline Nerve & Amplitude (mV) & Duration (ms) & CV m/s & Terminal latency (ms) & Area \\
\hline Ulnar R. & 1 & 9.5 & $44 / 69$ & 4 & 5 \\
\hline Ulnar L & 0.1 & 3.8 & $38 / 55$ & 3.7 & 0.2 \\
\hline MedianR & 0.5 & 8.4 & $56 / 68$ & 4.3 & 1.5 \\
\hline Median L. & 1.2 & 17.6 & $54 / 64$ & 6.6 & 10.5 \\
\hline Peroneal R. & 0.7 & 18.6 & $\begin{array}{c}\text { 24 (below fibular head- } \\
\text { popleteal fossa }\end{array}$ & 2.9 & - \\
\hline
\end{tabular}

These ENMG findings showed mainly axonal sensorimotor polyneuropathy with different degrees of affection of some nerves, more clearly seen in the lower limbs with partial motor conduction block in the left ulnar nerve (Table $1 \& 2$ ). 
Table 2: Site and value of the conduction block and electrophysiological data in left ulnar nerve.

\begin{tabular}{|c|c|c|c|c|c|c|c|c|c|c|}
\hline \multirow{2}{*}{ Level } & \multicolumn{2}{|c|}{ Amplitude (mV) } & \multirow{2}{*}{ R1 } & \multicolumn{2}{|c|}{ Area (mV ms) } & \multirow{2}{*}{$\mathbf{R 2}$} & \multicolumn{2}{|c|}{ Duration (ms) } & \multirow{2}{*}{$\mathrm{CV} \mathrm{m} / \mathrm{s}$} & \multirow{2}{*}{$\begin{array}{c}\text { Terminal latency } \\
\text { (ms) }\end{array}$} \\
\hline & Prox. & Distal & & Prox. & Distal & & Prox. & Distal & & \\
\hline $\begin{array}{l}\text { Below } \\
\text { eblow }\end{array}$ & 0.13 & 0.6 & $0.2(79 \%)$ & 0.23 & 2.08 & $0.1(88 \%)$ & 3.7 & 6.5 & $38 / 55$ & 3.7 \\
\hline
\end{tabular}

The right gastrocnemius muscle biopsy (stain H.E. and Van Gieson) showed various changes of the majority of epimysial, perimysial and endomysial arteries of medium and small sizes. Simultaneous atrophy of almost all muscle fibres with knots and chains of dark sarcolemmal striations are invisible and sarcolemmal nuclei disoriented. In transverse section, in some regions uniform small fibers with degenerative changes, dark cluster nuclei and mononuclear infiltration were also seen. In other regions of the sections increased variation of the diameter of muscle fibres, loss of few fibers, focal necrosis of some muscles with phagocytosis, the increased of connection tissue in the endomysium and cellular infiltration are evident. The skin biopsy from right lower leg, at the site of muscle biopsy, showed smoothing of the papillar layer and oedema with inflammatory mononuclear infiltration in the arterial and venous walls (Figure 1-6).

Cross section of biopsy of right gastrocnemius muscle (formolcalcium, Van Gieson stain) (Figure 1-6).
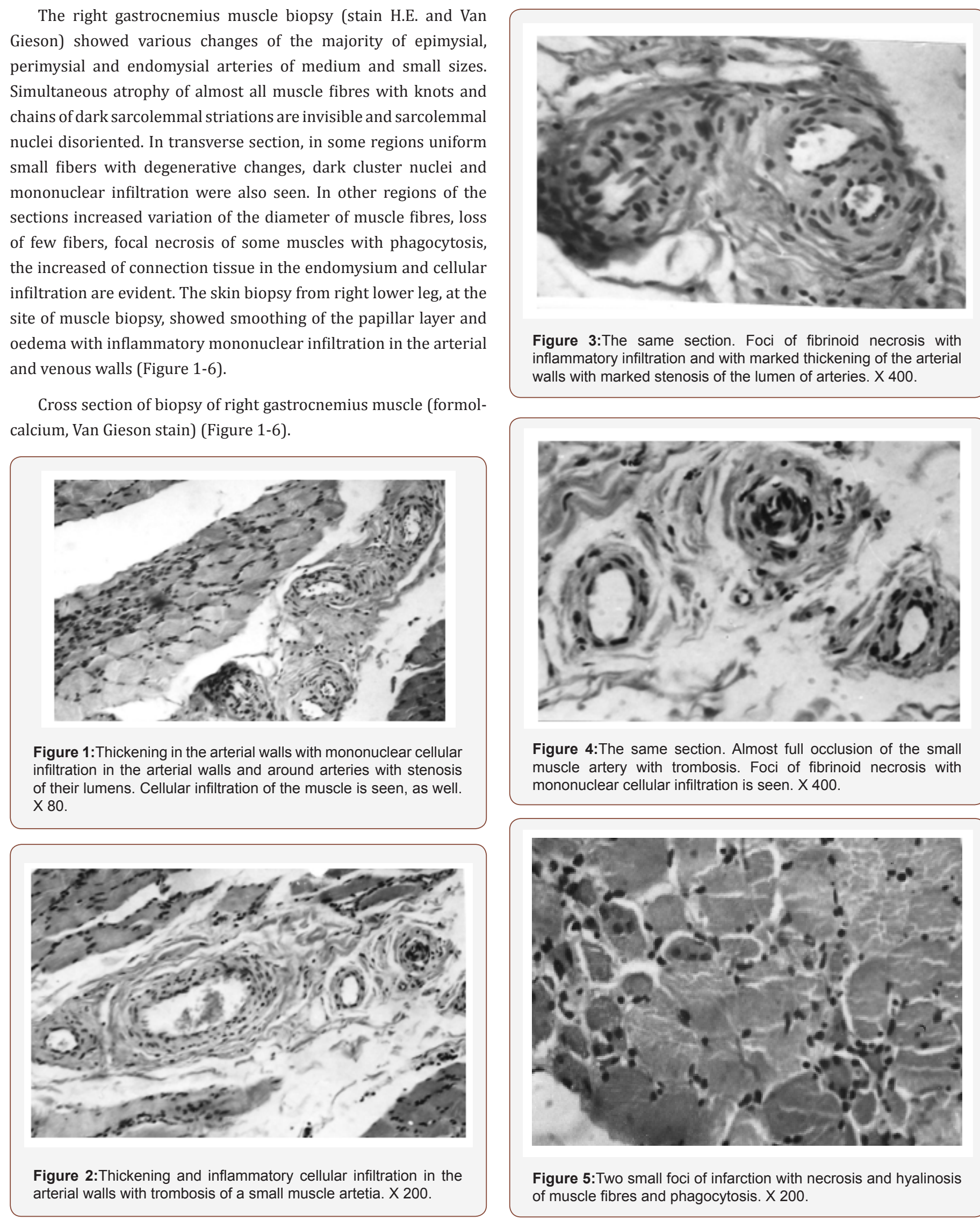

Figure 3:The same section. Foci of fibrinoid necrosis with inflammatory infiltration and with marked thickening of the arterial walls with marked stenosis of the lumen of arteries. X 400 .

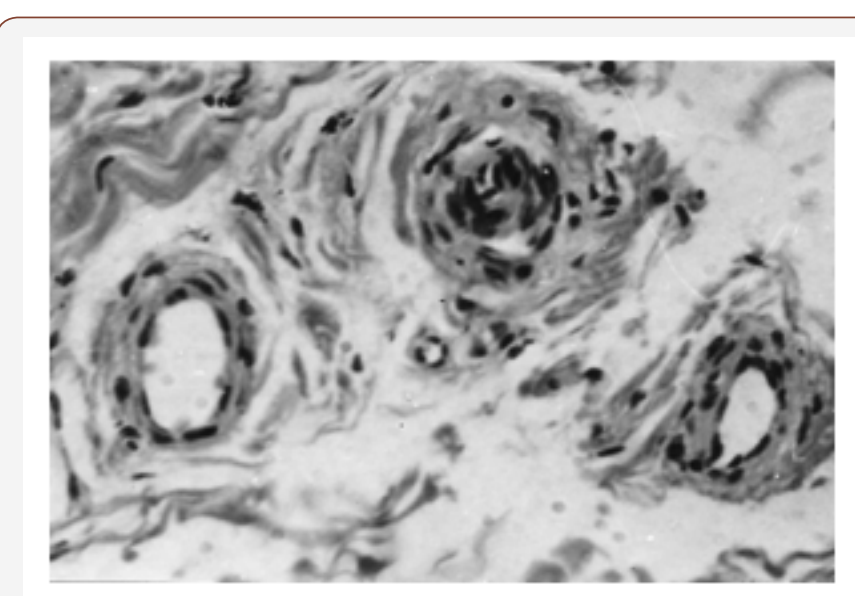

Figure 4:The same section. Almost full occlusion of the small muscle artery with trombosis. Foci of fibrinoid necrosis with mononuclear cellular infiltration is seen. X 400 .

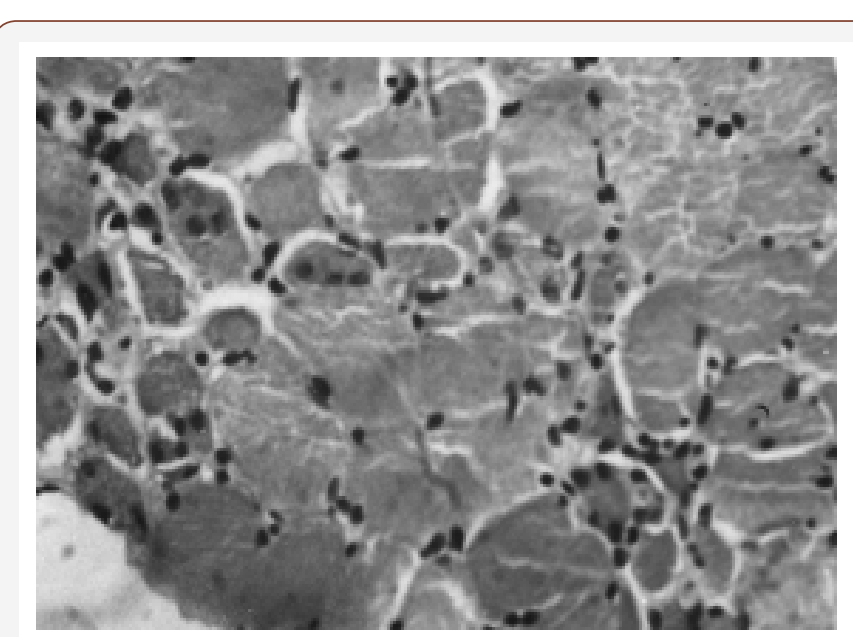

Figure 5:Two small foci of infarction with necrosis and hyalinosis of muscle fibres and phagocytosis. X 200 . 


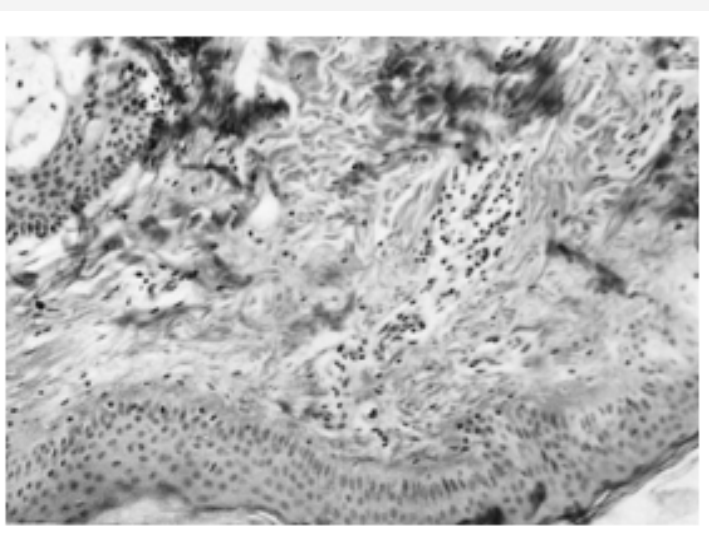

Figure 6:The skin biopsy (Van Gieson stain). The smooth papillar layer and oedema of dermis with inflammatory cellular infiltration in the vascular walls.

Thus the muscle biopsy findings were compatible with productive inflammatory vasculitis that resembled polyarteriitis nodosa with secondary neural muscular atrophy and nonspecific myositis. The patient was treated with intravenous infusions of dexamethasone $150 \mathrm{mg}$ daily for 5 days, then prednisolone $500 \mathrm{mg}$ daily for 5 days and later that he was treated with prednisolone 90 $\mathrm{mg} /$ day orally within intravenous invusion of cyclophosphamide $200 \mathrm{mg} /$ day for 15 days followed by cyclophosphamide $150 \mathrm{mg} /$ day orally and plasmapheresis. Sensory and motor disturbances decreased and the patient began walking with a cane. The skin swelling decreased, ESR and leucocytosis fell. But pronounced trophic and vegetative vascular disorders in toes were still present. The necrosis of the toes gradually progressed to dry gangrene. The patient was trransferred to a surgical department further moderate regression of the neurological symptoms but an increase in heart, lung, liver and kidney insufficiency. The patient suddenly died of thromboembolism of the pulmonary artery one year after disease began. This autopsy confirmed the diagnosis of periarteriitis nodosa.

\section{Conclusion}

1. The first symptoms of the disease (PN) indicated of peripheral nervous system involvement, namely, asymmetrical sensory neuropathy, without signs of systemic or polyorganic involvement.
2. Positive sensory disorders (pain, paresthesia, hyperpathia, hyperalgesia) in the legs were the main signs in the clinical picture in the early stages of the disease before the motor disorders appeared.

3. The asymmetrical sensory polyneuropathy progressed rapidly to a symmetrical sensorimotor peripheral polyneuropathy which preceded the clinical multisystem manifestastionsd.

4. ENMG and needle EMG data confirmed typical manifestation of sensorimotor peripheral polyneuropathy with characteristic axonal nerve lesions. But one of the peculiarities in the ischemic neuropathy in our patient was the signs of partial conduction block in the left ulnar nerve $[3,4]$.

5. In medical literature there are only a few descriptions of the appearances of conduction block in ischemic peripheral nerve lesion [1].

6. An unusual feature was the severe trophic disorder of the distal parts of the legs which did not respond to specific therapy an led to dry gangrene of the toes.

\section{Acknowledgement}

None.

\section{Conflict of Interest}

No conflict of interest.

\section{References}

1. Ropert A, Metral S (1990) Conduction block in neuropathies with necrotizing vasculitis. Muscle \& Nerve 13: 102-105.

2. Daube JR, Dyck P (1984) Neuropathy due to peripheral vascular discas. In: Dyk PJ, Thomas PK, Lambert EH (Eds.), Peripheral neuropathy. Philadelphia, Saunders, USA, pp. 1458-1478.

3. Feasby TE, Brown WF, Gilbert JL (1985) The pathological basis of conduction blockin human neiuropathies. Neurology Neurosurgery Psychiatry 48: 239-244.

4. Vital A, Vital C (1985) Poliarteritis nodosa and peripheral neuropathy. Intrasructural study of 13 cases. Acta Neuropathol. (Berl.) 67: 136-141.

5. Daniels I, Williams M, Worthingham C (1949) Muscle testing. Techniques of manual examination, $2^{\text {nd }}$ edn. Philadelphia, Saunders, USA. 